Check for updates

Cite this: RSC Adv., 2018, 8, 31608

\section{Using special Janus nanobelt as constitutional unit to construct anisotropic conductive array membrane for concurrently affording color- tunable luminescence and superparamagnetism $\uparrow$}

\author{
Xue Xi, ${ }^{a}$ Wensheng $\mathrm{Yu}{ }^{a}$ Qianli Ma, ${ }^{* a}$ Dan Li, ${ }^{a}$ Xiangting Dong, (D) *ab Jinxian Wang (D) ${ }^{a}$ \\ and Guixia Liu (DD ${ }^{a}$
}

\begin{abstract}
We have used electrospinning technology to fabricate a tri-functional nanobelt array membrane exhibiting tunable anisotropic electrical conduction, superparamagnetism and color-tunable luminescence by using a lab-made co-axis//single-axis spinneret and an aluminum drum collection device. Each one-dimensional (1D) Janus nanobelt is composed of luminescent-superparamagnetic bifunctional $\left[\mathrm{Fe}_{3} \mathrm{O}_{4} /\right.$ polymethyl methacrylate $(\mathrm{PMMA})] \mathrm{Q}\left[\mathrm{Tb}(\mathrm{BA})_{3}\right.$ phen/Eu(BA) ${ }_{3}$ phen/PMMA] coaxial nanobelt and conducting polyaniline (PANI)/PMMA nanobelt. Moreover, all Janus nanobelts are aligned in the same manner to generate a two-dimensional (2D) array film. The conductance along the length is much stronger than the conductance in the width (two perpendicular directions). Therefore, the array membrane has excellent anisotropic electrical conduction. The conduction ratio reaches $10^{8}$ times between the length and width of the Janus nanobelt array membrane, which is the highest conduction ratio between the two perpendicular directions for nanobelt materials reported internationally. Furthermore, we can modulate the degree of electrically conducting anisotropy of the samples by varying the amount of PANI. In addition, the Janus nanobelt array membrane is concurrently endowed by superior and adjustable superparamagnetism and photoluminescence. Importantly, the innovative philosophy and manufacturing technique of the new Janus nanobelt array membrane provide an easy way to prepare multifunctional nano-membranes.
\end{abstract}

Received 25th July 2018 Accepted 28th August 2018

DOI: $10.1039 / \mathrm{c} 8 \mathrm{ra06283h}$

rsc.li/rsc-advances

\section{Introduction}

Anisotropic conducting film (ACF) is a new type of electronic composite material, which exhibits one direction conduction and other direction insulation. ACFs are widely used in microelectronic packages, electrode conjuncture and other fields. ACFs can be divided into type I and type II ACFs in accordance with their conducting properties. In terms of type I ACFs, the directions along the film thickness and surface are conducting and insulating, respectively. The preparation technology of type I ACFs is advanced and widely used in the manufacture of modern electronic devices. Type II ACFs are single-layer or multi-layer thin films composed of parallel 1D conducting cells with different conductivities and different directions on the surface. These 1D conductors are mainly ultrafine fibers or long

${ }^{a}$ Key Laboratory of Applied Chemistry and Nanotechnology at Universities of Jilin Province, Changchun University of Science and Technology, Changchun 130022, China. E-mail: maqianlimail@cust.edu.cn; Fax: +86-0431-85383815

${ }^{b}$ Changchun University of Science and Technology, China. E-mail: xtdong@cust.edu. cn; Tel: +86-0431-85582574

$\dagger$ Electronic supplementary information (ESI) available. See DOI: 10.1039/c8ra06283h chains of molecules that guide electricity, which are separated by weak conductors such as air and/or interfaces. According to this particular structure, electrons move along the length of a 1D conducting element, but they are blocked vertically by air or an interface. Therefore, high conductivity can be observed along the length of the conducting element; however, low conductivity can be observed perpendicular to the length. Kovtyukhova et al. ${ }^{1}$ fabricated multiple layers of SWNTox/ polymer membranes by conventional layer and layer assembly; the vertical conductance was $10^{3}$ times lower than that along the length. Liu et $a .^{2}{ }^{2}$ prepared anisotropic conducting polymer films by electrospinning. The conductivity of the film along the vertical dimension was $10^{6}$ times lower than that along the length. Huang et al. ${ }^{3}$ fabricated an anisotropic conducting polymer membrane. The results showed that the resistivity along the stripe was 6 orders of magnitudes higher compared with the resistivity along the orthogonal MWCNT stripe. At present, many serious weaknesses of the type II ACFs remain; thus, type II ACFs cannot be used in industry and can only be studied in the laboratory. Much research needs to be done on type II ACFs before their large-scale industrialization or 
applications. Therefore, solving or alleviating these defects is an urgent research topic.

There are three ways to fabricate anisotropic conducting polymer nanomaterials. The first way is to orient the conducting material into the polymer and endow the polymer film with conducting anisotropy characteristics. ${ }^{4}$ The second way is to apply an anisotropic conducting belt as a synthetic unit; ${ }^{5,6}$ for example, Ma et al. used the electrospinning technique to prepare an array film made of conducting anisotropic Janus nanobelts and microbelts. Because of this unique structure, the conductivities along the perpendicular and length dimensions of the belts are up to $10^{8}$ times in disparity. The third way is to assemble the layered synthetic material into a strip 1D chain in a polymer. On this basis, several kinds of polymers in the strip chain and one dimensional chain technology, including shear assembly within magnetic field-induced self-assembly, guided by template assembly and nano filler and block copolymer collaborative assembly can be developed.

Electrospinning is the most convenient method to convert a viscous solution into a successive fiber with diameter ranging from micron to nanometer under high voltage. ${ }^{7-9}$ This technology has been popularized in the sectors of regenerative biology, electrochemical materials, optical fiber humidity sensors and filtration materials. At present, multifunctional nanomaterials are one of the hot topics in research. ${ }^{\mathbf{1 0}}$ For photoelectromagnetic nanomaterials, a previous study by our research team and other researchers demonstrated that dark colored magnetic $\mathrm{Fe}_{3} \mathrm{O}_{4}$ nanoparticles (NPs) and conducting PANI greatly decrease the photoluminescence properties of rare earth (RE) compounds as they are blended. ${ }^{11}$ Up to now, our scientific team has fabricated some multifunctional nanomaterials through electrospinning technology, which contain coaxial nanocables, ${ }^{\mathbf{1 2}}$ coaxial nanobelts, ${ }^{\mathbf{1 3}}$ Janus nanoribbons, ${ }^{\mathbf{1 4}}$ and Janus nanofibers. ${ }^{15,16}$ The $1 \mathrm{D}$ structures mentioned above can offer two independent partitions: RE compounds in one partition and $\mathrm{Fe}_{3} \mathrm{O}_{4}$ NPs and PANI in other partition. This special nanostructure can effectively separate RE compounds from $\mathrm{Fe}_{3} \mathrm{O}_{4}$ NPs and PANI, resulting in significant reduction in the luminescence of $\mathrm{RE}$ compounds by $\mathrm{Fe}_{3} \mathrm{O}_{4}$ NPs and PANI. The experiment also showed that while $\mathrm{Fe}_{3} \mathrm{O}_{4}$ NPs and PANI are mixed in one partition, $\mathrm{Fe}_{3} \mathrm{O}_{4}$ NPs cause PANI to be discontinuous in the nanofiber/nanobelt, thereby reducing the electrical conductivity of the nanomaterials. It is therefore necessary to further isolate the luminescent, magnetic and electrically conducting materials if the powerful photoluminescence performance and high conductance of the multifunctional nanomaterials are to be attained. To achieve this innovative academic concept, in this study, we developed a $\left\{\left[\mathrm{Fe}_{3} \mathrm{O}_{4} / \mathrm{PMMA}\right]\right.$ (a $\left[\mathrm{Tb}(\mathrm{BA})_{3} \text { phen/Eu(BA) }\right)_{3}$ phen/PMMA $\left.]\right\} / /[\mathrm{PANI} / \mathrm{PMMA}]$ (defined as [Magnetic@Luminescent]//Electrical, abbreviated as [M@Lum]//E) Janus nanobelt to implement three separate functional partitions included in one Janus nanobelt. The [M@Lum]//E Janus nanobelt was composed of two distinct sides comprising a $\left[\mathrm{Fe}_{3} \mathrm{O}_{4} / \mathrm{PMMA}\right] @\left[\mathrm{~Tb}(\mathrm{BA})_{3}\right.$ phen $/ \mathrm{Eu}(\mathrm{BA})_{3^{-}}$ phen/PMMA] coaxial nanobelt (magnetic-luminescent side) and a PANI/PMMA nanobelt (conducting side), and the Janus nanobelt successfully completed a variety of versatile asymmetric integrations. Furthermore, by using $\left\{\left[\mathrm{Fe}_{3} \mathrm{O}_{4} / \mathrm{PMMA}\right]\right.$ @ $\left[\mathrm{Tb}(\mathrm{BA})_{3} \text { phen/Eu(BA }\right)_{3}$ phen/PMMA $] / /[\mathrm{PANI} / \mathrm{PMMA}]$ as constitutional and conducting units, a $\left\{\left[\mathrm{Fe}_{3} \mathrm{O}_{4} / \mathrm{PMMA}\right] @\right.$ $\left[\mathrm{Tb}(\mathrm{BA})_{3}\right.$ phen/Eu(BA) ${ }_{3}$ phen/PMMA $] / / /$ PANI/PMMA $]$ Janus nanobelt array membrane (abbreviated as [M@Lum]//E JAM) was obtained via the parallel electrospinning technique. The array membrane combines high anisotropic electrical conduction, superparamagnetism and luminescence properties. To date, there has not been a report on such nanostructures. Moreover, to highlight the strength of [M@Lum]//E JAM, we also prepared samples of five different structures for comparison. The morphologies, structures, luminescence, superparamagnetism and electrically conducting anisotropy of the prepared samples were discussed, and we obtained some new conclusions.

\section{Experimental sections}

\section{Chemicals}

The chemicals are summarized in the ESI. $\dagger$

\section{Preparation of PMMA, $\mathrm{Fe}_{3} \mathrm{O}_{4} \mathrm{NPs}, \mathrm{Tb}(\mathrm{BA})_{3}$ phen and $\mathrm{Eu}(\mathrm{BA})_{3}$ phen compounds}

The preparation procedures of $\mathrm{PMMA}, \mathrm{Fe}_{3} \mathrm{O}_{4} \mathrm{NPs}, \mathrm{Tb}(\mathrm{BA})_{3}$ phen and $\mathrm{Eu}(\mathrm{BA})_{3}$ phen compounds are given in the ESI. $\dagger$

\section{Preparation of spinning solutions}

Three types of spinning solutions were used to synthesize [M@Lum]//E JAM, and their compositions are summarized in the ESI (Tables S1-S3). $\dagger$ To show the superior advantages of [M@Lum]//E JAM, five contrastive membranes were prepared including $\left\{\left[\mathrm{Fe}_{3} \mathrm{O}_{4} / \mathrm{PMMA}\right] @\left[\mathrm{~Tb}(\mathrm{BA})_{3} \text { phen/Eu(BA }\right)_{3}\right.$ phen/PMMA $\left.]\right\} / /$ [PANI/PMMA] Janus nanobelt non-array membrane (abbreviated as [M@Lum]//E JNM), $\left[\mathrm{Fe}_{3} \mathrm{O}_{4} / \mathrm{Tb}(\mathrm{BA})_{3} \text { phen/Eu(BA) }\right)_{3}$ phen/PMMA $] / /$ [PANI/PMMA] Janus nanobelt array membrane (abbreviated as $[\mathrm{M}-\mathrm{Lum}] / / \mathrm{E} \quad \mathrm{JAM}), \quad\left[\mathrm{Fe}_{3} \mathrm{O}_{4} / \mathrm{Tb}(\mathrm{BA})_{3} \text { phen/Eu(BA) }\right)_{3}$ phen/PMMA $] / /$ [PANI/PMMA] Janus nanobelt non-array membrane (abbreviated as $[\mathrm{M}$-Lum $] / / \mathrm{E} \quad \mathrm{JNM}), \quad \mathrm{Fe}_{3} \mathrm{O}_{4} / \mathrm{Tb}(\mathrm{BA})_{3}$ phen/Eu(BA) $)_{3}$ phen/PANI/ PMMA composite nanobelt array membrane (abbreviated as $\mathrm{M}$ Lum-E CAM), and $\mathrm{Fe}_{3} \mathrm{O}_{4} / \mathrm{Tb}(\mathrm{BA})_{3}$ phen/Eu(BA) ${ }_{3}$ phen/PANI/PMMA composite nanobelt non-array membrane (abbreviated as $\mathrm{M}$ Lum-E CNM). Among them, the constructive principles of the two contrastive samples M-Lum-E CAM and CNM are obtained from the ref. 11 and 17 . The spinning solutions used to prepare the contrastive samples and the components of the contrastive samples are summarized in the ESI $\dagger$ (Table S4 $\dagger$ ).

\section{Electrospinning process}

In the previous study, parallel spinnerets were used to fabricate Janus nanobelts with two isolated functional domains. The parallel spinnerets consisted of two bent stainless steel needles. ${ }^{5,14}$ In this study, we first designed and manufactured a new type of spinneret of co-axis//single-axis spinneret. Afterwards, novel and brand-new flexible peculiar-structured [coaxial nanobelt $] / /[$ nanobelt] Janus nanobelts with three isolated functional domains were prepared via electrospinning 

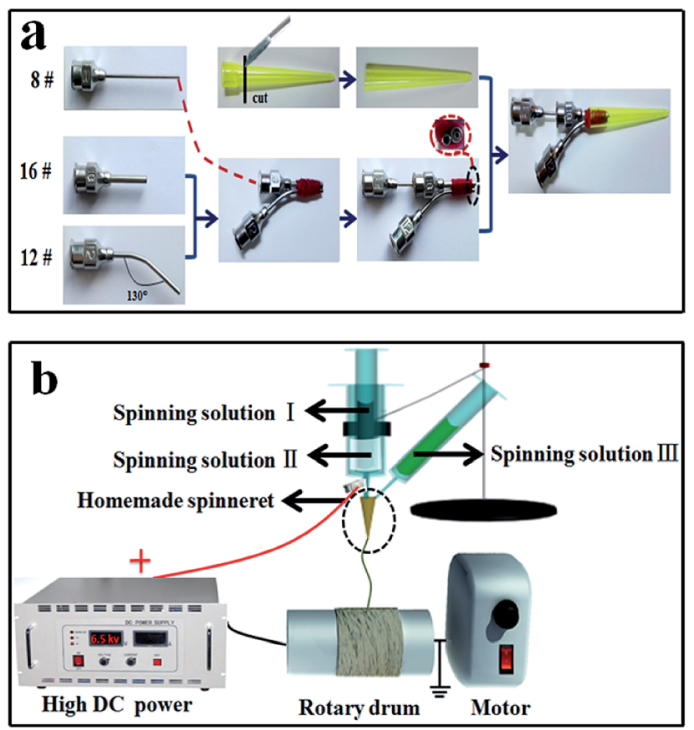

Fig. 1 Sketch maps of the preparation procedure for specially designed and installed co-axis//single-axis spinneret (a) and electrospinning equipment for fabricating [MaLum]//E JAM (b).

technology using the co-axis//single-axis spinneret. Thus, the co-axis//single-axis spinneret is more superior for the fabrication of nanobelts with more isolated functional domains. As shown in Fig. 1a, the specially made co-axis//single-axis spinneret consists of three different truncated stainless steel needles $(16 \#, 12 \#$ and $8 \#)$. The 16\# stainless steel needle and $8 \#$ stainless steel needle formed a coaxial spinneret, and a blended 12\# stainless steel needle was strapped to the coaxial spinneret abreast with a nylon string. A plastic jet nozzle was then placed on the top of the needles. The electrospinning apparatus for preparing[M@Lum]/E JAM is depicted in Fig. 1b. The spinning solutions I-III were separately poured into the three plastic syringes. To obtain an ordered array membrane, the experimental apparatus used an aluminum revolving drum as the nanobelt receiving device, and the rotational speed was moderated to $1500 \mathrm{rpm}$. The electrospinning conditions and electrospinning equipment for fabricating contrastive samples are listed in the ESI (please see Table $\mathrm{S} 4 \uparrow$ for more details).

\section{Results and discussion}

\section{Phase}

We characterized [M@Lum]/E JAM, five contrastive membranes and $\mathrm{Fe}_{3} \mathrm{O}_{4}$ NPs by X-ray diffractometry (Fig. 2). The XRD patterns of $\mathrm{Fe}_{3} \mathrm{O}_{4}$ NPs were in agreement with that of the cubic-structured of $\mathrm{Fe}_{3} \mathrm{O}_{4}$ (PDF 74-0748). The tests showed that all [M@Lum]//E JAM and contrastive samples contain $\mathrm{Fe}_{3} \mathrm{O}_{4}$ NPs.

\section{Morphology and internal structures}

The scanning electron microscopy (SEM) images of [M@Lum]//E JAM are shown in Fig. 3a, which shows that nearly all the Janus nanobelts are configured towards the same dimension. The

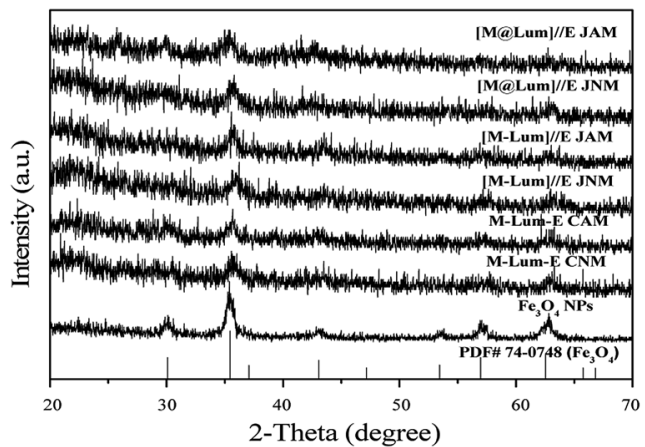

Fig. 2 XRD patterns of the samples with PDF standard card of $\mathrm{Fe}_{3} \mathrm{O}_{4}$.
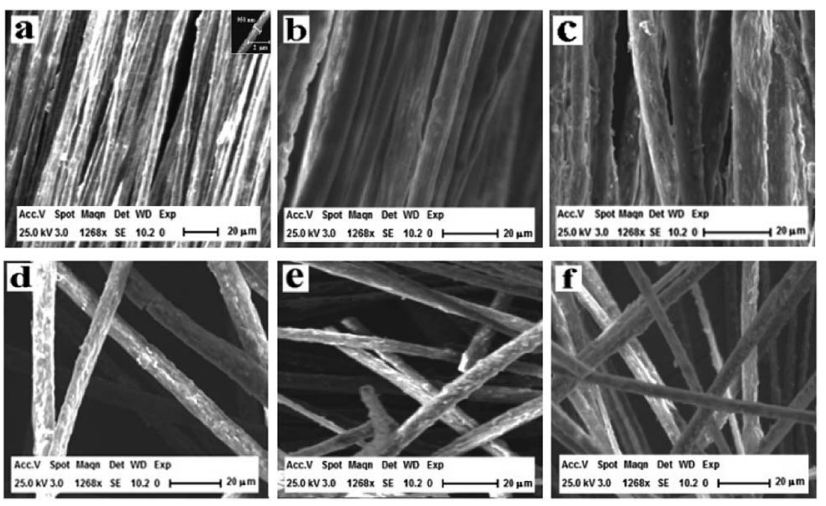

Fig. 3 SEM images of [M(aLum]//E JAM (a), [M-Lum]//E JAM (b), MLum-E CAM (c), [M@Lum]//E JNM (d), [M-Lum]//E JNM (e) and MLum-E CNM (f).

width of the [M@Lum]//E Janus nanobelts is $c a .6 .5 \mu \mathrm{m}$, and the thickness is shorter than $1 \mu \mathrm{m}$; the results show that nearly all of the broadsides of these nanobelts are face up, which may be because this state helps the nanobelts to deposit onto the rotary drum in a much more steady manner when they are in a lower position. On the basis of the biological microscopy (BM) image analysis, the internal structure of [M@Lum]/E Janus nanobelt can be observed. A clear Janus nanobelt structure can be observed in Fig. 4a, and one side of the Janus nanobelt is $\left[\mathrm{Fe}_{3} \mathrm{O}_{4} /\right.$ $\mathrm{PMMA}] @\left[\mathrm{~Tb}(\mathrm{BA})_{3} \text { phen/Eu(BA) }\right)_{3}$ phen/PMMA] coaxial nanobelt, which consists of a low-contrast shell and a dark-colored core; in contrast, the other side is PANI/PMMA. For further demonstration of the distinct Janus-type structure of the [M@Lum]/E Janus nanobelts, energy dispersive spectroscopy (EDS) line scan analysis was conducted (Fig. $4 \mathrm{~b}$ ); $\mathrm{Tb}, \mathrm{Eu}, \mathrm{Fe}$ and $\mathrm{S}$ represent $\mathrm{Tb}(\mathrm{BA})_{3}$ phen, $\mathrm{Eu}(\mathrm{BA})_{3}$ phen, $\mathrm{Fe}_{3} \mathrm{O}_{4}$ and $\mathrm{PANI}$, respectively. Elemental Fe exists in the middle domain of the $\left[\mathrm{Fe}_{3} \mathrm{O}_{4} / \mathrm{PMMA}\right]$ @ $\left[\mathrm{Tb}(\mathrm{BA})_{3} \text { phen/Eu(BA }\right)_{3}$ phen/PMMA $]$ coaxial nanobelt. In addition, we found that only elemental $\mathrm{Tb}$ and $\mathrm{Eu}$ are dispersed on both sides of the coaxial nanobelt, and the other half side of the [M@Lum]/E Janus nanobelt is PANI/PMMA. Through SEM, BM, EDS line-scan analyses, we inferred that [M@Lum]/E JAM has been successfully prepared.

Fig. $3 \mathrm{~b}$ and $\mathrm{c}$ show SEM images of [M-Lum]/E JAM and MLum-E CAM, respectively. As can be seen in Fig. 3b and c, the 

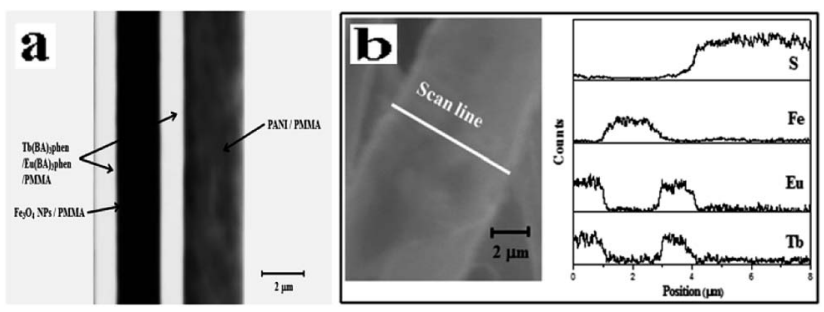

Fig. 4 BM image (a) and EDS line-scan analysis (b) of a single [MaLum]//E Janus nanobelt.

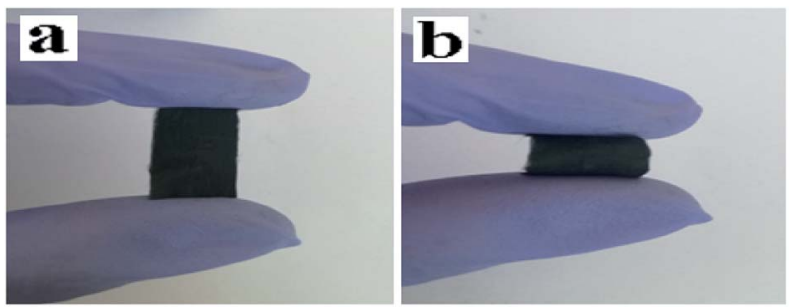

Fig. 5 Digital photos of [MaLum]//E JAM: (a) an unbent and (b) bent Janus nanobelt array membrane.

widths of each single [M-Lum]//E Janus nanobelt and M-Lum-E composite nanobelt are $c a .7 .5 \mu \mathrm{m}$ and $8 \mu \mathrm{m}$, respectively. In a further survey, [M-Lum]//E Janus nanobelts and M-Lum-E composite nanobelts are ordered in all samples. The SEM images of [M@Lum]//E Janus nanobelt non-array, [M-Lum]//E Janus nanobelt non-array and M-Lum-E composite nanobelt non-array are shown in Fig. 3d, e and f, respectively, whereas the Janus nanobelts and composite nanobelts are out-of-order. The widths of the independent nanobelt in [M@Lum]//E Janus nanobelt non-array, [M-Lum]//E Janus nanobelt non-array and M-Lum-E composite nanobelt non-array are $c a .8 \mu \mathrm{m}, 7 \mu \mathrm{m}$ and $5.5 \mu \mathrm{m}$, respectively. Fig. 5 shows typical digital photos of [M@Lum]//E JAM, and we can observe from the photos that the membrane can be easily bent and folded. This is very important for future applications of materials in flexible devices.

\section{Fluorescent property}

Luminescence performances of [M@Lum]//E JAM were studied. Color-tunable luminescence was obtained by modifying the ratios of $\mathrm{Eu}(\mathrm{BA})_{3}$ phen to $\mathrm{Tb}(\mathrm{BA})_{3}$ phen (samples $\left[\mathrm{S}_{\mathrm{a} 2} @ \mathrm{~S}_{\mathrm{b} x}\right] / / \mathrm{S}_{\mathrm{c} 2}, x$ $=1-5$ ) when the mass percentage of PANI to PMMA was fixed at $30 \%$ and the mass ratio of $\mathrm{Fe}_{3} \mathrm{O}_{4}$ to PMMA was fixed at $1: 1$. Based on previous experimental investigations, ${ }^{18}$ we chose $290 \mathrm{~nm}$ ultraviolet light as the excitation (abbreviated as EX) source for $\mathrm{Tb}(\mathrm{BA})_{3}$ phen and $\mathrm{Eu}(\mathrm{BA})_{3}$ phen compounds; under this ultraviolet light, the emission (abbreviated as EM) spectra of [M@Lum]//E JAM exhibited peaks at 490 and $545 \mathrm{~nm}$, which were ascribed to the ${ }^{5} \mathrm{D}_{4} \rightarrow{ }^{7} \mathrm{~F}_{J}(J=6,5)$ energy level transitions of $\mathrm{Tb}^{3+}$, and the peaks at 592 and $616 \mathrm{~nm}$ were due to the ${ }^{5} \mathrm{D}_{0} \rightarrow$ ${ }^{7} \mathrm{~F}_{J}(J=1,2)$ energy level transitions of $\mathrm{Eu}^{3+}$, as seen in Fig. 6 (left). The test results showed that as the mass ratio of $\mathrm{Eu}(\mathrm{BA})_{3}$ phen to $\mathrm{Tb}(\mathrm{BA})_{3}$ phen decreased, the green fluorescence $\mathrm{EM}$ of $\mathrm{Tb}^{3+}$ ions at 490 and $545 \mathrm{~nm}$ gradually increased, and the
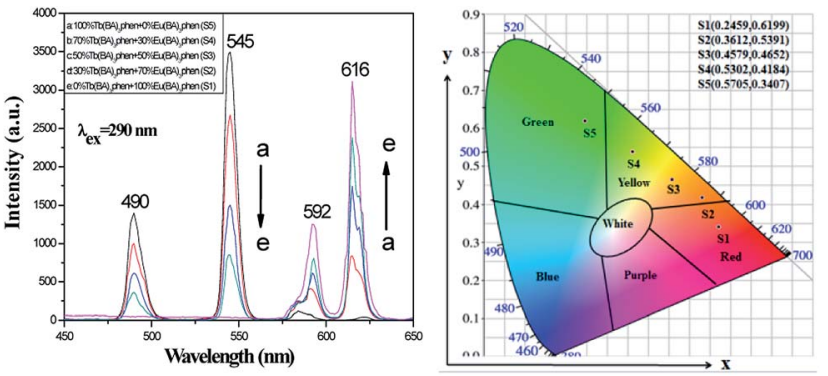

Fig. 6 EM spectra (left) and $\mathrm{CIE}$ chromaticity diagram (right) of [M(aLum]//E JAM containing various mass ratios of $\mathrm{Eu}(\mathrm{BA})_{3}$ phen to $\mathrm{Tb}(\mathrm{BA})_{3}$ phen.

orange and red fluorescence $\mathrm{EM}$ at 592 and $615 \mathrm{~nm}$ of $\mathrm{Eu}^{3+}$ ions slowly decreased. Fig. 6 shows the CIE coordinates for [M@Lum]//E JAM excited by $290 \mathrm{~nm}$, and the experiment verified that after adjusting the mass ratios of $\mathrm{Eu}(\mathrm{BA})_{3}$ phen compounds to $\mathrm{Tb}(\mathrm{BA})_{3}$ phen compounds, the fluorescence color of the samples can be adjusted from red to green.

In addition, [M@Lum]//E JAM containing various amounts of $\mathrm{Fe}_{3} \mathrm{O}_{4}$ NPs was fabricated to detect the influence of the addition of various amounts of $\mathrm{Fe}_{3} \mathrm{O}_{4}$ NPs (samples $\left[\mathrm{S}_{\mathrm{ax}} @ \mathrm{~S}_{\mathrm{b}_{3}}\right] / /$ $\left.\mathrm{S}_{\mathrm{c} 2}, x=1-4\right)$ on the fluorescence performances of [M@Lum]//E JAM when the mass ratio of $\mathrm{Eu}(\mathrm{BA})_{3}$ phen to $\mathrm{Tb}(\mathrm{BA})_{3}$ phen was set at $1: 1$ and the mass percentage of PANI to PMMA was fixed at $30 \%$, as shown in Fig. 7 a. It was discovered that as the content of $\mathrm{Fe}_{3} \mathrm{O}_{4}$ NPs increased, the EM intensities of [M@Lum]//E JAM decreased. The results demonstrated the light absorption of $\mathrm{Fe}_{3} \mathrm{O}_{4}$ NPs. To explain the effect of $\mathrm{Fe}_{3} \mathrm{O}_{4}$ NP content on the fluorescence intensity, sketch maps of EX and EM lights in [M@Lum]//E JAM containing various mass ratios of $\mathrm{Fe}_{3} \mathrm{O}_{4}$ NPs to PMMA are shown in Fig. 8. Fig. 7b shows the absorption spectrum of $\mathrm{Fe}_{3} \mathrm{O}_{4}$ NPs. It was observed that $\mathrm{Fe}_{3} \mathrm{O}_{4}$ NPs can
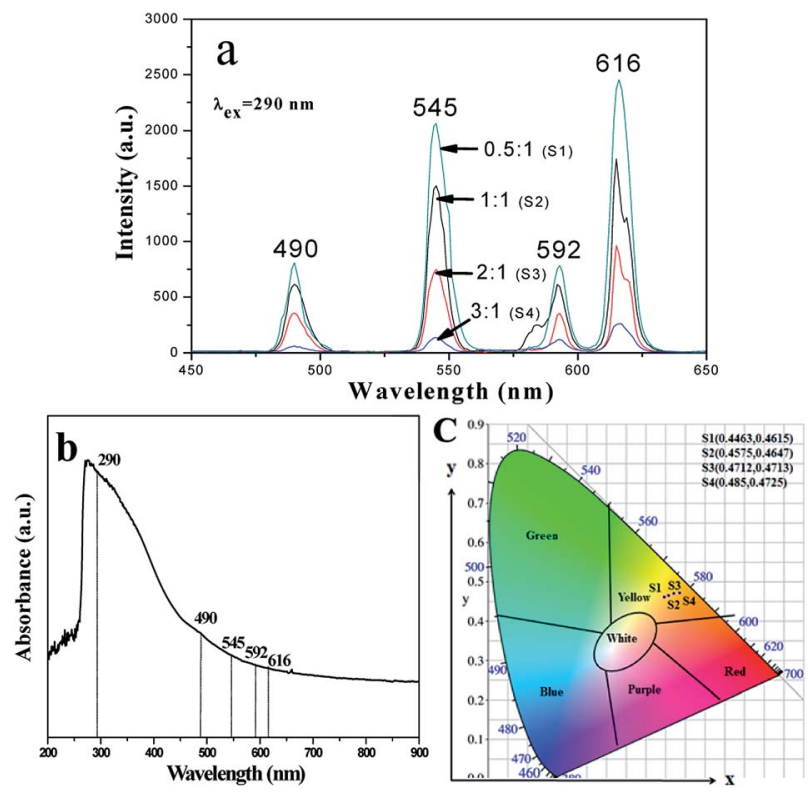

Fig. 7 EM spectra (a) and CIE chromaticity diagram (c) of [M@Lum]//E JAM containing various mass ratios of $\mathrm{Fe}_{3} \mathrm{O}_{4}$ NPs to PMMA; UV-vis absorption spectrum of $\mathrm{Fe}_{3} \mathrm{O}_{4} \mathrm{NPs}(\mathrm{b})$. 

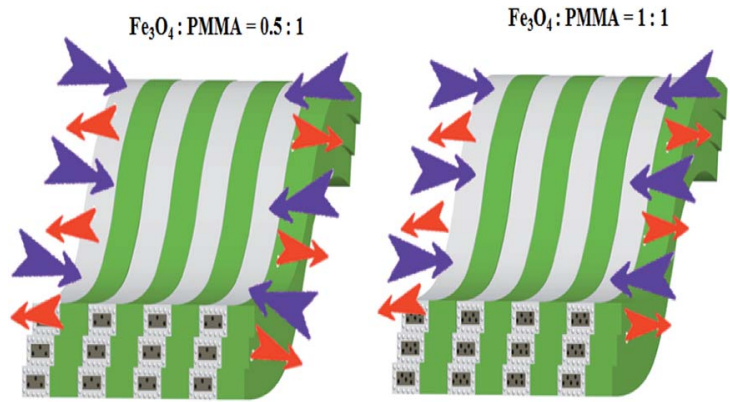

$\mathrm{Fe}_{3} \mathrm{O}_{1}: \mathrm{PMMA}=2: 1$

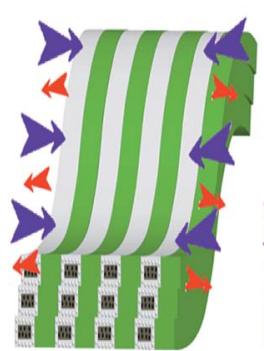

$\mathrm{Fe}_{3} \mathrm{O}_{4}: \mathrm{PVMMA}=3: 1$
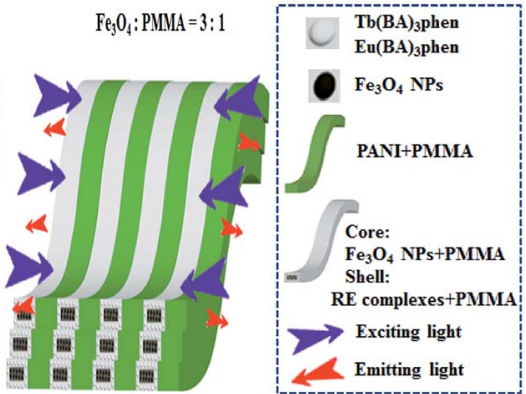

Fig. 8 Sketch maps of EX and EM light in [M@Lum]//E JAM containing various mass ratios of $\mathrm{Fe}_{3} \mathrm{O}_{4} \mathrm{NPs}$ to PMMA.

absorb visible light from $200 \mathrm{~nm}$ to $900 \mathrm{~nm}$. Thus, the intensities of the EX and EM peaks decreased as the EX and EM lights were absorbed and weakened by $\mathrm{Fe}_{3} \mathrm{O}_{4}$ NPs. Fig. 7c demonstrates the CIE chromaticity diagram of [M@Lum]//E JAM with various $\mathrm{Fe}_{3} \mathrm{O}_{4}$ amounts under EX of $290 \mathrm{~nm}$ ultraviolet light. The results showed that the EM color of [M@Lum]/E JAM shifted to red with the addition of more $\mathrm{Fe}_{3} \mathrm{O}_{4}$ NPs, and this elaborated the theory that $\mathrm{Fe}_{3} \mathrm{O}_{4}$ has powerful light absorption to the light at lower wavelengths. With an increase in the amount of $\mathrm{Fe}_{3} \mathrm{O}_{4} \mathrm{NPs}$, the EM color of [M@Lum]//E JAM shifted toward red. This disclosed the fact that $\mathrm{Fe}_{3} \mathrm{O}_{4}$ has more powerful light absorption for light of lower wavelengths.

Meanwhile, the fluorescence properties of [M@Lum]//E JAM with various amounts of PANI (samples $\left[\mathrm{S}_{\mathrm{a} 2} @ \mathrm{~S}_{\mathrm{b} 3}\right] / / \mathrm{S}_{\mathrm{c} x}, x=1-4$ ) are studied when the mass ratio of $\mathrm{Eu}(\mathrm{BA})_{3}$ phen to $\mathrm{Tb}(\mathrm{BA})_{3}$ phen and $\mathrm{Fe}_{3} \mathrm{O}_{4}$ NPs to PMMA are fixed at $1: 1$. As the PANI content increases, the EM intensities tend to decrease due to the light absorption of dark-colored PANI, as demonstrated in Fig. 9a. To illustrate the influence of different amounts of PANI on fluorescence, a schematic of EX and EM light in the array membrane with various percentages is shown in Fig. 10. As manifested in Fig. 9b, PANI strongly absorbs light in the range of $270-360 \mathrm{~nm}, 380-450 \mathrm{~nm}$ and $500-800 \mathrm{~nm}$. Therefore, the EX and EM lights are absorbed by the PANI, resulting in reduced intensity of the EX and EM peaks. The CIE chromaticity coordinates for [M@Lum]//E JAM are shown in Fig. 9c. It is observed that with the introduction of PANI, the EM color of [M@Lum]//E JAM shifts to green, which is due to gradual intense absorption of red light by PANI.

Five contrastive samples were also prepared, and their fluorescence performances were in contrast with the fluorescence performance of [M@Lum]/E JAM. As seen from Fig. 11, compared with [M@Lum]//E JAM, [M@Lum]//E JNM exhibited
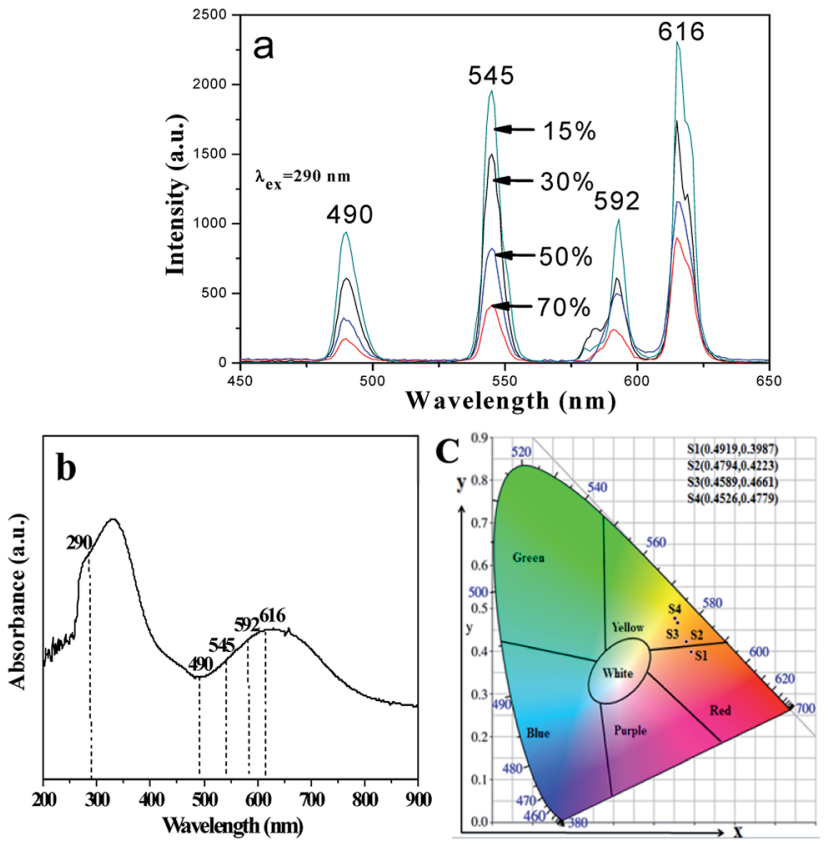

Fig. 9 EM spectra (a) and CIE chromaticity diagram (c) of [M@Lum]//E JAM containing various mass percentages of PANI to PMMA; UV-vis absorption spectrum of PANI (b).

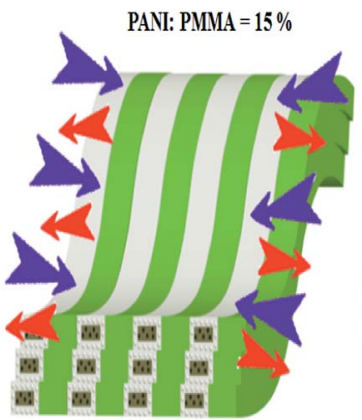

PANE: PIIMA $=50 \%$

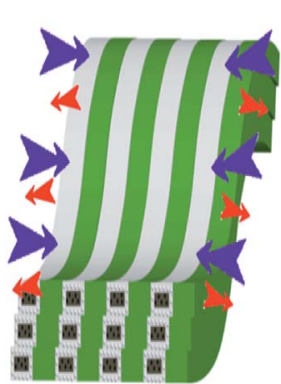

Fig. 10 Sketch maps of the EX and EM light in [M@Lum]//E JAM containing various mass percentages of PANI to PMMA.

slightly decreased fluorescence intensity. The results also indicated that other samples had weaker fluorescence intensity when they contained the same content of raw materials. Sketch maps of the EX and EM lights in [M@Lum]//E JAM and contrastive samples are shown in Fig. 12; the figure showed that the surface of [M@Lum]//E JAM was dense because it consisted of parallel Janus nanobelts. The EX light experienced difficulty 


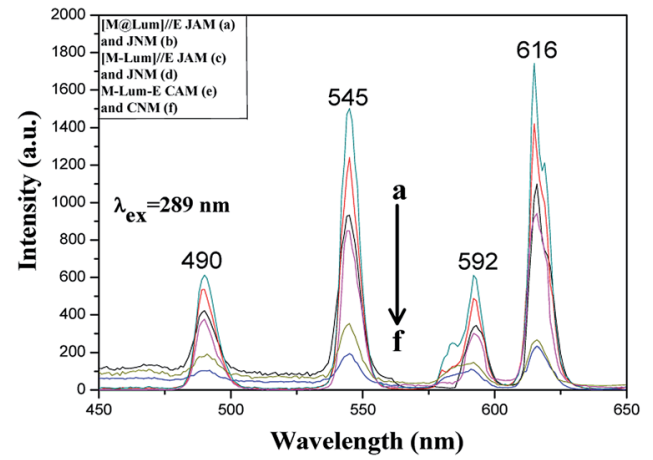

Fig. 11 EM spectra of [M@Lum]//E JAM (a) and JNM (b), [M-Lum]//E JAM (c) and JNM (d), M-Lum-E CAM (e) and CNM (f).

while penetrating the upper layer and reached the lower Janus nanobelts; thus, the EM light was mainly emitted from the upper Janus nanobelts without reduction. As for [M@Lum]//E JNM, the disordered Janus nanobelts led to a loose membrane surface. In this situation, part of the EX light penetrated the loose upper voids to the lower Janus nanobelts. This led to the absorption of the EX light by the upper Janus nanobelts; thus, the EX light intensity weakened. In the same manner, the EX light must go through the gap in the upper layers, which is also absorbed and attenuated. In the end, the fluorescence intensity
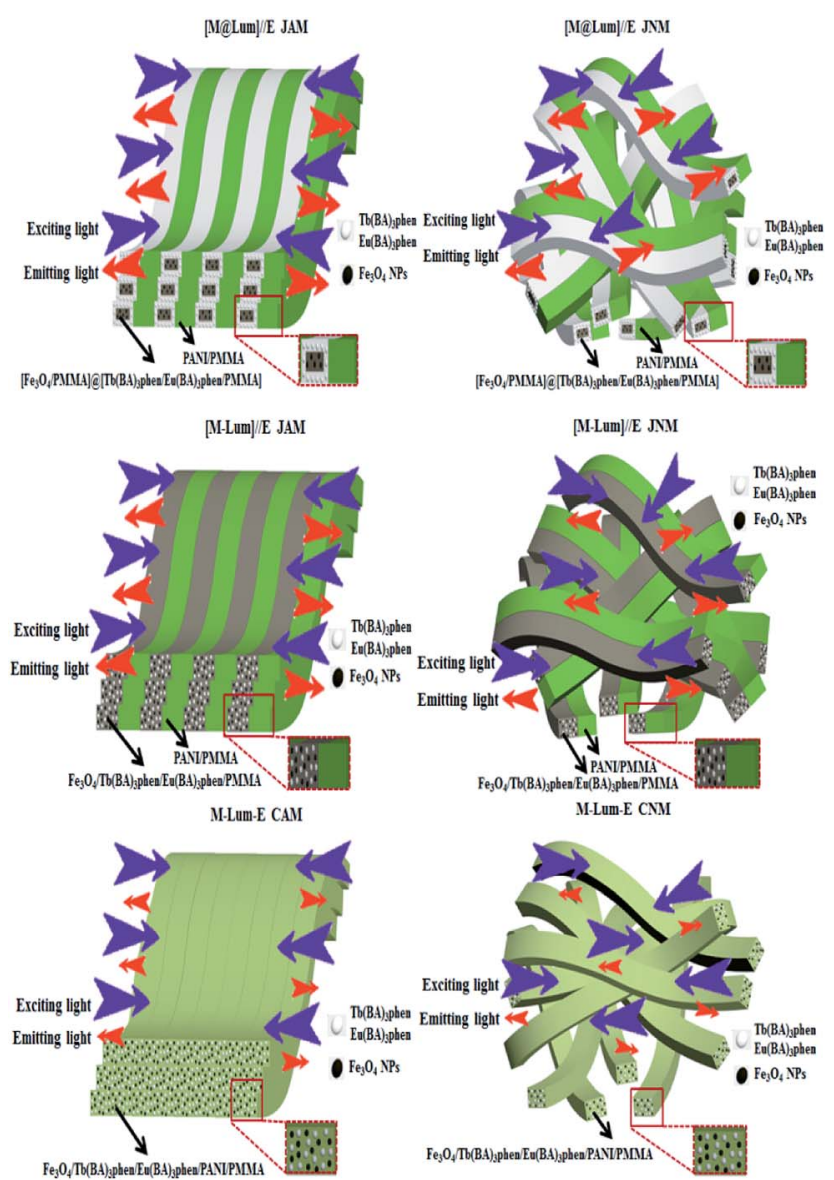

Fig. 12 Sketch maps of EX and EM light in [MaLum]//E JAM and contrastive samples. of [M@Lum]/E JNM was lower than that of [M@Lum]//E JAM. Likewise, [M-Lum]//E JAM exhibited more powerful fluorescence intensity than [M-Lum]//E JNM, and M-Lum-E CAM exhibited stronger fluorescence intensity than M-Lum-E CNM. As is seen in Fig. 11, [M@Lum]//E JAM and JNM exhibited significantly stronger luminescence intensities than other contrastive samples. As for [M@Lum]//E JAM and JNM, the constitutional unit is the coaxial nanobelt//nanobelt ([Magnetic@Luminescent]//Electrical) type Janus nanobelt, which achieves efficient isolation of PANI and $\mathrm{Fe}_{3} \mathrm{O}_{4}$ NPs from $\mathrm{RE}$ compounds, due to which the EX and EM lights absorbed by PANI and $\mathrm{Fe}_{3} \mathrm{O}_{4}$ NPs are significantly reduced. For [M-Lum]//E JAM and JNM, the constitutional unit is the nanobelt// nanobelt ([Magnetic-Luminescent]//Electrical) Janus nanobelt, which also benefits the isolation of RE compounds and $\mathrm{Fe}_{3} \mathrm{O}_{4}$ NPs from PANI; nevertheless, the RE compounds and $\mathrm{Fe}_{3} \mathrm{O}_{4}$ NPs are still randomly distributed together in the $\mathrm{Fe}_{3} \mathrm{O}_{4} / \mathrm{Tb}(\mathrm{BA})_{3^{-}}$ phen/Eu(BA) ${ }_{3}$ phen/PMMA nanobelts, due to which $\mathrm{Fe}_{3} \mathrm{O}_{4}$ NPs absorb and weaken the EX and EM lights. In terms of M-Lum-E CAM and CNM, the constitutional unit is the (MagneticLuminescent-Electrical) composite nanobelt, in which the RE compounds, $\mathrm{Fe}_{3} \mathrm{O}_{4}$ NPs and PANI are averagely dispersed as a whole in $\mathrm{Fe}_{3} \mathrm{O}_{4} / \mathrm{Tb}(\mathrm{BA})_{3}$ phen/Eu(BA) $)_{3}$ phen/PANI/PMMA nanobelts, with the result that the deep-colored $\mathrm{Fe}_{3} \mathrm{O}_{4}$ NPs and PANI strongly absorb and weaken the EX and EM lights. The above testing results demonstrate that the [M@Lum]//E Janus-structured nanobelts with three independent partitions have better performance than [M-Lum]//E Janus-structured nanobelts with two independent partitions and M-Lum-E composite nanobelts without any partition.

\section{Superparamagnetic property}

Fig. 13 shows typical hysteresis loops of [M@Lum]//E JAM containing various mass ratios of $\mathrm{Fe}_{3} \mathrm{O}_{4}$ NPs to PMMA. As shown in Fig. 13, the samples exhibit superparamagnetic performance. The saturation magnetizations of the samples are summarized in Table 1, and the test results show that the saturation magnetization of [M@Lum]/E JAM increases as more $\mathrm{Fe}_{3} \mathrm{O}_{4}$ NPs are introduced into the $\mathrm{Fe}_{3} \mathrm{O}_{4} /$ PMMA core; this indicates that the superparamagnetism of the samples can be adjusted. In addition, as seen in Table 1, the saturation magnetizations of contrastive samples and [M@Lum]/E JAM

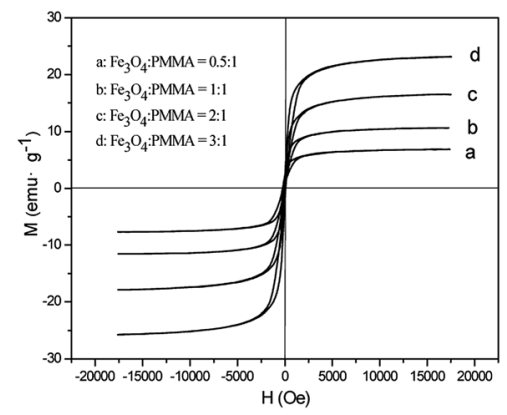

Fig. 13 Hysteresis loops of [M@Lum]//E JAM containing various mass ratios of $\mathrm{Fe}_{3} \mathrm{O}_{4}$ NPs to PMMA. 
Table 1 Saturation magnetizations of [M@Lum]//E JAM containing various mass ratios of $\mathrm{Fe}_{3} \mathrm{O}_{4}$ to $\mathrm{PMMA}$ and contrastive samples

Saturation magnetization Samples $\left(M_{\mathrm{s}}\right) /\left(\mathrm{emu} \mathrm{g}^{-1}\right)$

$\begin{array}{lr}{\left[\mathrm{S}_{\mathrm{a} 1} @ \mathrm{~S}_{\mathrm{b} 3}\right] / / \mathrm{S}_{\mathrm{c} 2}\left(\mathrm{Fe}_{3} \mathrm{O}_{4}: \text { PMMA }=0.5: 1\right)} & 5.22 \\ {\left[\mathrm{~S}_{\mathrm{a} 2} @ \mathrm{~S}_{\mathrm{b} 3}\right] / / \mathrm{S}_{\mathrm{c} 2}\left(\mathrm{Fe}_{3} \mathrm{O}_{4}: \text { PMMA }=1: 1\right)} & 9.67 \\ {\left[\mathrm{~S}_{\mathrm{a} 3} @ \mathrm{~S}_{\mathrm{b} 3}\right] / / \mathrm{S}_{\mathrm{c} 2}\left(\mathrm{Fe}_{3} \mathrm{O}_{4}: \text { PMMA }=2: 1\right)} & 15.83 \\ {\left[\mathrm{~S}_{\mathrm{a} 4} @ \mathrm{~S}_{\mathrm{b} 3}\right] / / \mathrm{S}_{\mathrm{c} 2}\left(\mathrm{Fe}_{3} \mathrm{O}_{4}: \text { PMMA }=3: 1\right)} & 23.19 \\ {[\mathrm{M} @ L \mathrm{Lum}] / / \mathrm{E} \mathrm{JNM}} & 9.71 \\ {[\mathrm{M} \text {-Lum] }] / \text { E JAM }} & 9.53 \\ {[\mathrm{M}-\mathrm{Lum}] / / \mathrm{E} \mathrm{JNM}} & 9.76 \\ \text { M-Lum-E CAM } & 10.11 \\ \text { M-Lum-E CNM } & 9.34\end{array}$

$\left(\left[\mathrm{S}_{\mathrm{a} 2} @ \mathrm{~S}_{\mathrm{b} 3}\right] / / \mathrm{S}_{\mathrm{c} 2}\right)$ are very close when they have similar components and constituents. Based on the above luminescence and magnetism test analyses, [M@Lum]//E JAM and contrastive samples have the same superparamagnetism, but [M@Lum]/E JAM has larger luminescence intensity than the contrastive samples, indicating that [M@Lum]//E JAM exhibits better performance.

\section{Electrical conduction analysis}

Fig. 14 shows the test methods for the electrical conduction of array (Fig. 14a and b) and non-array membranes (Fig. 14c and d). As shown in Fig. 14, two pieces of soldering tin $(0.45 \mathrm{~cm}$ in width and $1 \mathrm{~cm}$ in length) are placed on the specimen (the specimen is cut into $1 \mathrm{~cm} \times 1 \mathrm{~cm}$ ); then, the two pins of the Hall effect measurement system are connected to the two pieces of soldering tin. For the array membranes, the conductance along the length and width of the nanobelts in the membranes can be determined. For the non-array membranes, the conductance along a random dimension (equivalent to length) and the
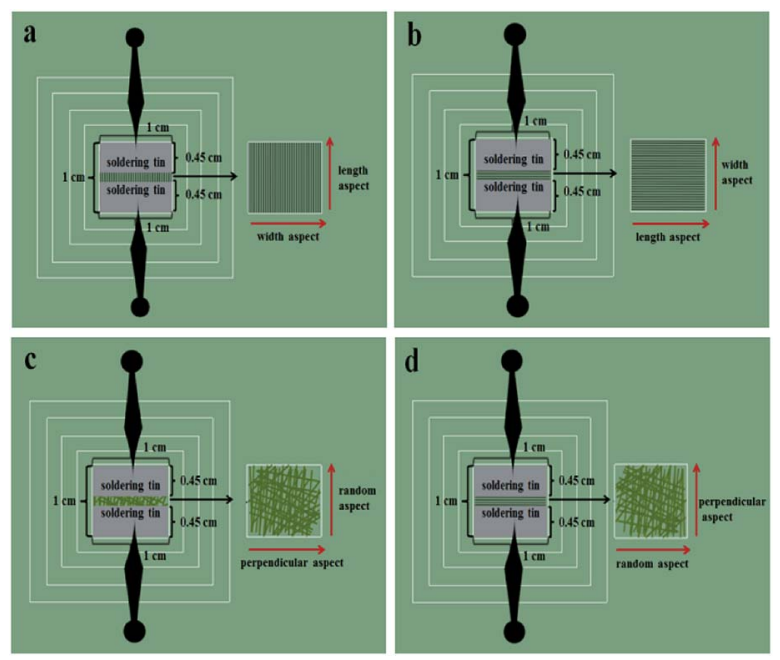

Fig. 14 Test methods for electrical conduction along the length (a) and width (b) of the nanobelts in array membrane and along the random dimension (c) and perpendicular dimension (d) in non-array membrane. corresponding perpendicular dimension (equivalent to width) can be measured. The electrical properties of [M@Lum]//E with various amounts of PANI (samples $\left[\mathrm{S}_{\mathrm{a} 2} @ \mathrm{~S}_{\mathrm{b} 3}\right] / / \mathrm{S}_{\mathrm{c} x}, x=1-4$ ) are measured, and the determined conductance values and ratios between length and width are listed in Table 2. From Table 2, it can be seen that the conductance of these array membranes can be tuned by adjusting the mass percentage of PANI to PMMA, and the conductance along the length of the [M@Lum]//E Janus nanobelts in the array membrane sharply increases from $6.24 \times$ $10^{-7} \mathrm{~S}$ to $4.41 \times 10^{-2} \mathrm{~S}$ when the mass percentage of PANI to PMMA changes from $15 \%$ to $70 \%$. The conductance along the width dimension retains $c a$. 10 orders of magnitude, resulting in a high conductance ratio between length and width. Besides, this shows that the conductance along the length is $c a$. 8 orders of magnitude higher than that along the width when the mass percentage of PANI is fixed at $70 \%$, which proves that [M@Lum]//E JAM has strong anisotropy in electrical conduction; as far as we know, the volume is the largest conductance ratio between the two perpendicular directions for nanobelt membrane. The results show that [M@Lum]//E JAM is electrically conducting only along the length and insulative along the width, and the Janus nanobelts have electrically conducting anisotropy that can be adjusted by varying the PANI content.

Conductance and electrically conducting anisotropy of the [M@Lum]/E JAM and contrastive samples were comparatively studied when the mass percentage of PANI to PMMA was set to $30 \%$, as seen in Table 3 and Fig. 15. In Table 3, the ratio of length to width in [M-Lum]//E JAM is $1.34 \times 10^{4}$, which is similar to that of [M@Lum]//E JAM $\left(7.12 \times 10^{4}\right)$, implying that both have strong anisotropy. In contrast, composite nanobelt array membranes only have weak electrically conducting anisotropy $\left(5.43 \times 10^{1}\right)$. Fig. 15 shows the sketch maps for the electrical properties of [M@Lum]//E JAM and contrastive samples. In [M@Lum]/E JAM and [M-Lum]/E JAM, electrons can flux along the length of the Janus nanobelts without obstacles and are obstructed along the width owing to the presence of insulative [M@Lum] or [M-Lum] nanobelts, resulting in a higher conductance ratio between the two perpendicular dimensions of the two types of membranes. Compared with [M@Lum]//E JAM and [M-Lum]//E JAM, M-Lum-E CAM has significantly lower electrically conducting anisotropy. On the one hand, the electrically conducting unit is the PANI/PMMA nanobelt in [M@Lum]//E JAM and [M-Lum]//E JAM, which achieves efficient isolation of insulative RE compounds and $\mathrm{Fe}_{3} \mathrm{O}_{4}$ NPs from conducting PANI and guarantees the high conductance along the length of the Janus nanobelts. Nevertheless, the electrically conducting unit is $\mathrm{Fe}_{3} \mathrm{O}_{4} / \mathrm{Tb}(\mathrm{BA})_{3}$ phen/ $\mathrm{Eu}(\mathrm{BA})_{3}$ phen/PANI/PMMA nanobelts in M-Lum-E CAM, which prevents the building of a continuous PANI conducting network and reduces the conductance of the composite nanobelts. As seen in Table 3, the conductance values along the length of [M@Lum]//E JAM and [M-Lum]//E JAM are higher than that along the length of M-Lum-E CAM. On the other hand, there are no effective insulative units among the nanobelts in M-Lum-E CAM; only the interfaces between the composite nanobelts can be used as a less effective insulating medium and thus, the conductance along the width of the nanobelts in M-Lum-E CAM 
Table 2 Conductance volumes and anisotropy degree of [M@Lum]//E JAM

\begin{tabular}{lllll}
\hline Samples & Length direction (S) & Width direction (S) & $\begin{array}{l}\text { Ratio of length } \\
\text { direction to width direction }\end{array}$ & Anisotropic degree \\
\hline$\left[\mathrm{S}_{\mathrm{a} 2} @ \mathrm{~S}_{\mathrm{b} 3}\right] / / \mathrm{S}_{\mathrm{c} 1}$ (PANI/PMMA: $\left.15 \%\right)$ & $6.24 \times 10^{-7}$ & $2.48 \times 10^{-10}$ & $2.52 \times 10^{3}$ & Medium \\
{$\left[\mathrm{S}_{\mathrm{a} 2} @ \mathrm{~S}_{\mathrm{b} 3}\right] / / \mathrm{S}_{\mathrm{c} 2}(\mathrm{PANI} / \mathrm{PMMA}: 30 \%)$} & $8.33 \times 10^{-6}$ & $1.17 \times 10^{-10}$ & $7.12 \times 10^{4}$ & $\mathrm{Medium}$ \\
{$\left[\mathrm{S}_{\mathrm{a} 2} @ \mathrm{~S}_{\mathrm{b} 3}\right] / / \mathrm{S}_{\mathrm{c} 3}$ (PANI/PMMA: 50\%) } & $5.75 \times 10^{-4}$ & $3.24 \times 10^{-10}$ & $1.77 \times 10^{6}$ & Strong \\
{$\left[\mathrm{S}_{\mathrm{a} 2} @ \mathrm{~S}_{\mathrm{b} 3}\right] / / \mathrm{S}_{\mathrm{c} 4}$ (PANI/PMMA: 70\%) } & $4.41 \times 10^{-2}$ & $1.79 \times 10^{-10}$ & $2.46 \times 10^{8}$ & Very strong
\end{tabular}

Table 3 Conductance volumes of [M@Lum]//E JAM and contrastive samples

\begin{tabular}{lllll}
\hline Samples & Length direction (S) & Width direction (S) & $\begin{array}{l}\text { Ratio of length } \\
\text { direction to width direction }\end{array}$ & Anisotropic degree \\
\hline [M@Lum]//E JAM & $8.33 \times 10^{-6}$ & $1.17 \times 10^{-10}$ & $7.12 \times 10^{4}$ & Strong \\
[M@Lum]//E JNM & $7.07 \times 10^{-7}$ & $6.28 \times 10^{-7}$ & 1.13 & None \\
[M-Lum]/E JAM & $7.42 \times 10^{-6}$ & $5.51 \times 10^{-10}$ & $1.34 \times 10^{4}$ & Strong \\
[M-Lum]/E JNM & $9.02 \times 10^{-8}$ & $9.67 \times 10^{-8}$ & 0.93 & None \\
M-Lum-E CAM & $5.59 \times 10^{-7}$ & $1.03 \times 10^{-8}$ & $5.43 \times 10^{1}$ & Weak \\
M-Lum-E CNM & $4.47 \times 10^{-8}$ & $4.22 \times 10^{-8}$ & 1.06 & None
\end{tabular}

is higher than that along the width in [M@Lum]//E JAM and [MLum]//E JAM. In brief, the insulating performance along the width of the nanobelts in M-Lum-E CAM is not strong enough.

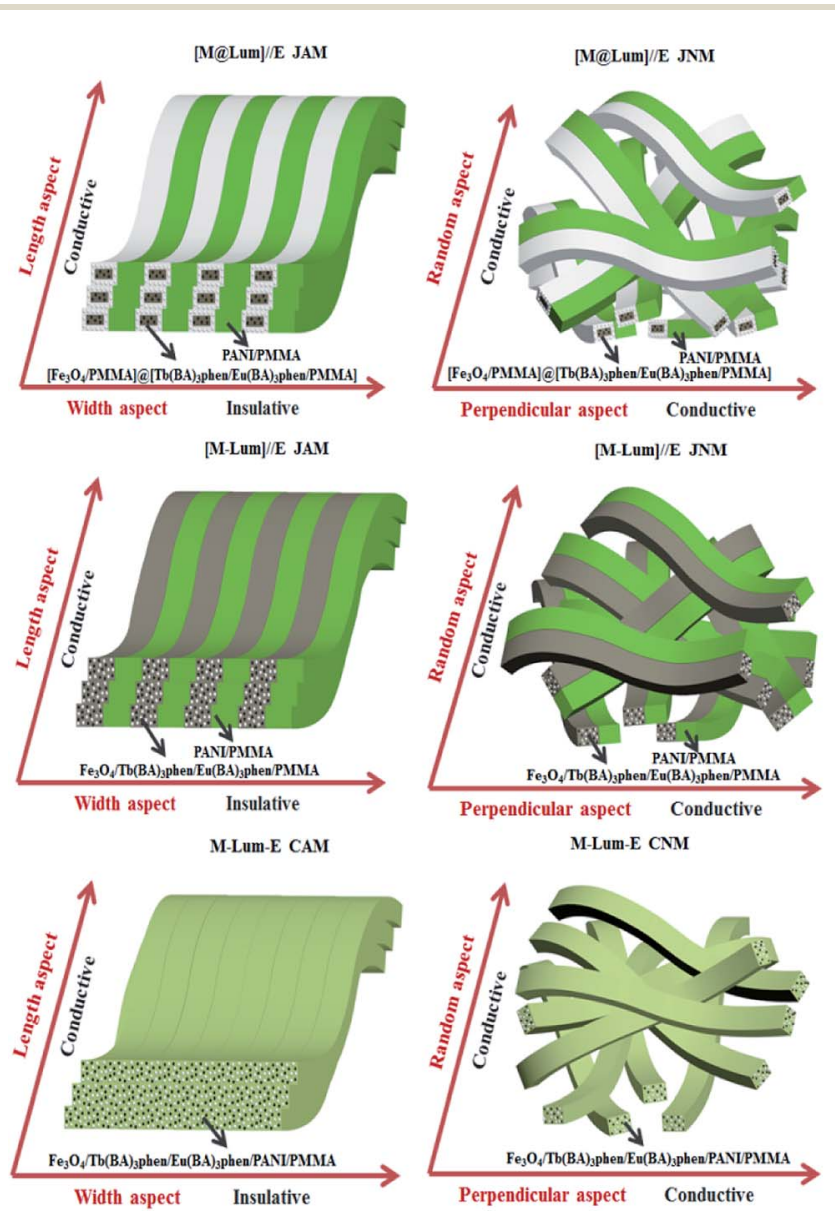

Fig. 15 Sketch maps of electrical conduction in [M@Lum]//E JAM and contrastive samples.
As shown in Table 3, the non-array membranes have negligible electrically conducting anisotropy. As show in Fig. 15, because the nanobelts in [M-Lum]//E JNM and M-Lum-E CNM are disordered in arrangement, the direction of current flow is disordered, which leads to electrically conducting isotropy of these non-array membranes. Under these circumstances, because electrical conduction exists in all directions of the membrane, the volume of conductance decreases in a particular dimension. From what has been discussed above, we can conclude that two key requirements must be met in the preparation of highly conducting anisotropic films. The other is that the insulator unit will be used and inserted into the insulating direction of the array film to further block the movement of electrons.

In combination with the above fluorescence analysis, superparamagnetism and electrically conducting anisotropy, we are pleased to discover that the fluorescence intensity of [M@Lum]//E JAM is much stronger than that of [M-Lum]//E JAM when they have similar magnetism and electrically conducting anisotropy, proving that the new [M@Lum]//E JAM have better luminescent-superparamagnetic-electrical properties than $[\mathrm{M}$ Lum]//E JAM. The [M@Lum]//E Janus nanobelts with three isolated partitions are superior in structuring multifunctional nanomaterials as compared with the [M-Lum]//E Janus nanobelts with two isolated partitions and M-Lum-E composite Janus nanobelts without any partition.

\section{Thermal stability analysis}

The TG curve for [M@Lum]//E JAM is shown in Fig. 16. [M@Lum]//E JAM loses about 1.31\% of its initial weight when the temperature is increased from room temperature to $130{ }^{\circ} \mathrm{C}$ due to volatilization of surface absorbed water. With the continuous increase in temperature, organic compounds such as $\mathrm{Tb}(\mathrm{BA})_{3}$ phen, $\mathrm{Eu}(\mathrm{BA})_{3}$ phen, PMMA and PANI start to 


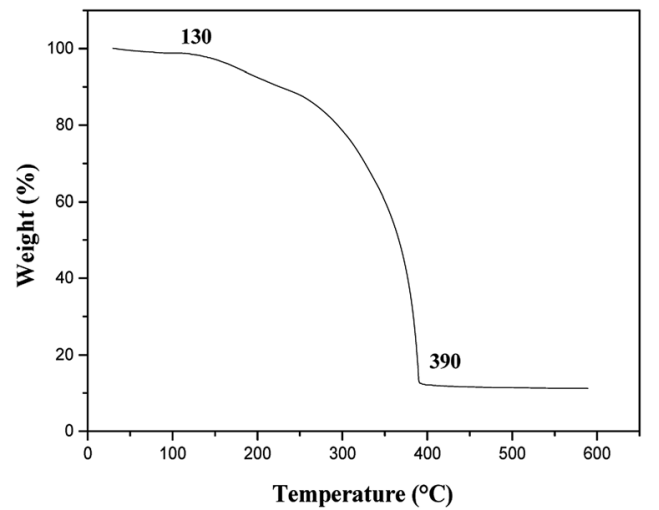

Fig. 16 TG curve of [MaLum]//E JAM.

decompose, and the decomposition process is completed at $390^{\circ} \mathrm{C}$. The total weight loss percentage is $87.83 \%$. These results show that [M@Lum]//E JAM can be safely applied below $130{ }^{\circ} \mathrm{C}$.

\section{Conclusions}

In summary, we have established a new method to construct novel and brand-new flexible peculiar-structured [coaxial nanobelt $] /[$ nanobelt $]$ Janus nanobelts, which are very different from already-reported [nanobelt] $/ /[$ nanobelt] structure type Janus nanobelts, via electrospinning technology using a newlydesigned and manufactured co-axis//single-axis spinneret. With the aid of the peculiar-structured [coaxial nanobelt]/[nanobelt] Janus nanobelts, luminescent, magnetic and electrical substances are mutually isolated, and mutual interferences among them can be further reduced, due to which excellent trifunctionalities of luminescence, electricity and magnetism are concurrently integrated into the Janus nanobelts. Additionally, from the microcosmic angle, partition of only two isolated functional domains can be realized by using the alreadyreported common 1D Janus nanobelt; nevertheless, partition of three isolated functional domains rather than two isolated functional domains is successfully realized in the new $1 \mathrm{D}$ Janus nanobelt reported in this study. Therefore, the novel Janus nanobelts can also be extended to assemble various three functions to realize multi-functionalization without reducing mutual interferences. Furthermore, the peculiar-structured [coaxial nanobelt] $] /[$ nanobelt] Janus nanobelts are used to build anisotropic conductive array membranes. Because $\left[\mathrm{Fe}_{3} \mathrm{O}_{4} /\right.$ PMMA]@[Tb(BA) $)_{3}$ phen/Eu(BA) ${ }_{3}$ phen/PMMA $]$ as insulative units are inserted into the insulative portion of the array membranes, which further blocks the movement of electrons, the largest conductivity ratio between the length and width directions of the nanobelts in the array membrane reaches up to eight orders of magnitude, which is the highest conductivity ratio between the two perpendicular dimensions for nanobelt membranes. Moreover, the electrically conducting anisotropy of Janus nanobelt array membranes can be tuned by adjusting the amount of PANI. Besides, the Janus nanobelt array membrane is simultaneously endued with tunable superparamagnetism and color-tunable fluorescence. The design philosophy and the construction technique for the novel Janus nanobelts and array membranes can afford a facile approach for the fabrication of Janus nanobelts and array membranes with other tri- or multifunctionalities.

\section{Conflicts of interest}

There are no conflicts of interest to declare.

\section{Acknowledgements}

This work was financially supported by National Natural Science Foundation of China $(51573023,50972020)$, Natural Science Foundation of Jilin Province (20170101101JC, 20180520011JH), Industrial Technology Research and Development Project of Jilin Province Development and Reform Commission (2017C052-4), Science and Technology Research Planning Project of the Education Department of Jilin Province during the 13th five-year plan period (JJKH20170608KJ, JJKH20181122KJ), and Innovative Foundation (XJJLG-2017-04) and Youth Foundation (XQNJJ-2016-01, XQNJJ-2017-17) of Changchun University of Science and Technology.

\section{References}

1 N. I. Kovtyukhova and T. E. Mallouk, J. Phys. Chem. B, 2005, 109, 2540-2545.

2 C. Feng, K. Liu, J. S. Wu, L. Liu, J. S. Cheng, Y. Y. Zhang, Y. H. Sun, Q. Q. Li, S. S. Fan and K. L. Jiang, Adv. Funct. Mater., 2010, 20, 885-891.

3 J. R. Huang, Y. T. Zhu, W. Jiang and Q. X. Tang, ACS Appl. Mater. Interfaces, 2014, 6, 1754-1758.

4 W. A. D. Heer, A. Chatelain and D. Ugarte, Science, 1995, 270, 1179-1181.

5 Q. L. Ma, J. X. Wang, X. T. Dong, W. S. Yu and G. X. Liu, Adv. Funct. Mater., 2015, 25, 2436-2443.

6 Q. L. Ma, W. S. Yu, X. T. Dong, M. Yang, J. X. Wang and G. X. Liu, Sci. Rep., 2015, 5, 14583.

7 L. Han, M. M. Pan, Y. Lv, Y. T. Gu, X. F. Wang, D. Li, Q. L. Kong and X. T. Dong, J. Mater. Sci.: Mater. Electron., 2015, 26, 677-684.

8 X. Xi, Q. L. Ma, M. Yang, X. T. Dong, J. X. Wang, W. S. Yu and G. X. Liu, J. Mater. Sci.: Mater. Electron., 2014, 25, 4024-4032.

9 J. Tian, Q. L. Ma, W. S. Yu, X. T. Dong, Y. Yang, B. Zhao, J. X. Wang and G. X. Liu, New J. Chem., 2017, 41, 1398313992.

10 X. B. Li, Q. L. Ma, J. Tian, X. Xi, D. Li, X. T. Dong, W. S. Yu, X. L. Wang, J. X. Wang and G. X. Liu, Nanoscale, 2017, 9, 18918-18930.

11 K. Lun, Q. L. Ma, M. Yang, X. T. Dong, Y. Yang, J. X. Wang, W. S. Yu and G. X. Liu, J. Mater. Sci.: Mater. Electron., 2015, 26, 5994-6003.

12 Q. L. Ma, J. X. Wang, X. T. Dong, W. S. Yu and G. X. Liu, Chem. Eng. J., 2013, 222, 16-22.

13 H. Shao, Q. L. Ma, X. T. Dong, W. S. Yu, M. Yang, J. X. Wang and G. X. Liu, Phys. Chem. Chem. Phys., 2015, 17, 2184521855. 
14 Q. L. Ma, J. X. Wang, X. T. Dong, W. S. Yu and G. X. Liu, Nanoscale, 2014, 6, 2945-2952.

15 X. Xi, J. X. Wang, X. T. Dong, Q. L. Ma, W. S. Yu and G. X. Liu, Chem. Eng. J., 2014, 254, 259-267.

16 X. Xi, Q. L. Ma, X. T. Dong, D. Li, W. S. Yu, J. X. Wang and G. X. Liu, J. Mater. Sci.: Mater. Electron., 2018, 29, 7119-7129.
17 K. Lun, Q. L. Ma, M. Yang, X. T. Dong, Y. Yang, J. X. Wang, W. S. Yu and G. X. Liu, Chem. Eng. J., 2015, 279, 231-240.

18 J. Tian, Q. L. Ma, X. T. Dong, M. Yang, Y. Yang, J. X. Wang, W. S. Yu and G. X. Liu, J. Mater. Sci.: Mater. Electron., 2015, 26, 8413-8420. 